\title{
Physics of top
}

\author{
C.-P. Yuan* \\ Department of Physics \& Astronomy \\ Michigan State University \\ East Lansing, MI 48824 \\ USA
}

E-mail: yuanepa.msu.edu

I will briefly review the physics of top quark at high energy colliders. A new discovery of singletop event at the Fermilab Tevatron is expected. At the CERN Large Hadron Collider, detailed top quark properties can be measured and new physics ideas in which top quark plays a special role can be tested. I will also discuss a few phenomenological methods for analyzing experimental data to study top quark interactions.

International Workshop on Top Quark Physics

January 12-15, 2006

Coimbra, Portugal

* Speaker. 


\section{Introduction}

Prior to the discovery of top quark in 1995, a wide range of its mass was predicted [1], which signals our ignorance about the origin of mass. Hence, the breaking mechanisms of electroweak symmetry (for generating $W$ and $Z$ boson masses) and flavor symmetry (for generating a wide spectrum of fermion masses) remain to be two of the major mysteries in the elementary particle physics. Nevertheless, through the process of "guessing" what the top quark mass is, we learned that only experimental data has the final say about the mother Nature; the interaction between experimentalists and theorists is essential for the advance of science; and theorists should not give up any probable ideas. In this talk, I will discuss a few theory ideas in which top quark plays a special role such that studying the interaction of top quark might help revealing the mechanism of electroweak symmetry breaking (EWSB) and flavor symmetry breaking mechanisms. I will also discuss a few phenomenological methods for analyzing experimental data to study top quark interactions.

\section{Impact of $m_{t}$ measurement}

First, let us briefly review what we know so far about the top quark. Its mass $\left(m_{t}\right)$ has been measured with better accuracy around $174 \mathrm{GeV}$ from studying the top quark pair events produced via quark and gluon fusion processes at the Fermilab Tevatron [2]. It is quite challenging to measure $m_{t}$ from the $b j j$ invariant mass to be better than a couple of $\mathrm{GeV}$ at the Tevatron and the CERN Large Hadron Collider (LHC), because of the uncertainty in jet energy resolution (related to underlying hadronic activities in the event) and the limited accuracy in current theory calculations when the accuracy in the $m_{t}$ measurement $\left(\delta m_{t}\right)$ is required to be less than the width of top quark. Other means for measuring $m_{t}$ are: studying the fraction of longitudinal polarization of the $W$ boson (or the transverse momentum $\left(p_{T}\right)$ distribution of the lepton) and the invariant mass distribution of $b$ and $\ell$ in the decay of top quark $t \rightarrow b W(\rightarrow \ell v)$ [3]. How well do we need to know about $m_{t}$ in order to test the Standard Model (SM) beyond the tree level? At the Tevatron Run-II, $\delta m_{t} \sim 2-3 \mathrm{GeV}$, and it is no longer a dominant error in precision test until $\delta M_{W}$ is reduced to about $20 \mathrm{MeV}$. At the LHC, $\delta m_{t} \sim 1.5 \mathrm{GeV}$, which is about the same as the top quark width $\left(\Gamma_{t}\right)$, and the counterpart in the measurement of $M_{W}$ needs to be about $10 \mathrm{MeV}$. Again, we see that $\delta m_{t}$ is not the dominant theoretical error in testing the SM from comparing its predictions to precision data.

At the future International Linear Collider (ILC), $\delta m_{t}$ can be reduced to a couple of hundred $\mathrm{MeV}$ (of the order of $\Lambda_{Q C D}$ ) from studying the top quark pair production at threshold (from the measurement of total cross section, peak of transverse momentum distribution, and the forwardbackward asymmetry), and around $500 \mathrm{MeV}$ from directly reconstructing the mass of top quark produced at continuum [4].

\section{Top quark decay}

Because its mass is at the weak scale, top quark will decay like a bare quark (without first forming hadrons) so that we could study its spin property in addition to measure its decay branching ratios. In the SM, top decays into $b W$ mode almost $100 \%$ of time. With new physics coupling to top 
quark, many new decay channels become possible, such as $t \rightarrow b H^{+}, \tilde{t} \chi_{0}, c \gamma, c Z, c g, c h^{0}$ predicted in the Minimal Supersymmetric Standard Model (MSSM). Thus, it is important to measure the decay branching ratio (BR) of $t \rightarrow b W$. Unfortunately, in the SM, the CKM matrix element $V_{t b}$ is so much larger than $V_{t s}$ and $V_{t d}$ that the ratio of $\mathrm{BR}(t \rightarrow b W)$ to $\mathrm{BR}(t \rightarrow b q)$ cannot effectively measure the magnitude of the $W-t-b$ coupling. Moreover, the total decay width of the top quark cannot be accurately measured from the $b j j$ invariant mass distribution in top decays, for the experimental uncertainty due to jet energy resolution is much larger than $\Gamma_{t}[5]$.

It is however possible that new physics effect might not change the value of $\mathrm{BR}(t \rightarrow b W)$, for not having additional new light fields with mass less than $m_{t}$, but could still modify $\Gamma_{t}$ when the interaction of $t-b-W$ is strongly modified. Hence, we need to directly measure the interaction strength of $t-b-W$ coupling. This can be done by studying single-top production rates initiated from weak charged current processes. In particular, the SM tree level t-channel single-top inclusive production rate $\left(\sigma_{t}\right)$ is proportional to the decay width of top quark. Hence, $\sigma_{t}$ can be used to determine the CKM matrix element $V_{t b}$ in the SM, and to determine the partial decay width $\Gamma(t \rightarrow$ $b W)$ [6]. When combining the measurement of $\mathrm{BR}(t \rightarrow b W)$ from $t \bar{t}$ events and $\Gamma(t \rightarrow b W)$ from t-channel singe-top event, one can determine the life-time of top quark from their ratio.

\section{Single-top production}

At the Tevatron and the LHC, single-top events can be produced from t-channel, s-channel and $W t$ associate production processes [7]. Their production rates can be largely modified by new physics interactions, either with new heavy resonances, such as $W^{\prime}, Z^{\prime}, H^{ \pm}, \pi^{\prime}$, or with flavor changing neutral currents (FCNC), such as $t c Z, t u Z, t c g, t c \gamma$, or with new flavor changing charged currents (FCC), such as $t s W, t d W, t b H^{+}$. It turns out that the s-channel mode is sensitive to charged resonances. The t-channel mode is more sensitive to FCNC and new interactions. The $t W$ mode is a more direct measure of top quark coupling to $W$ and a down-type (down, strange, bottom) quark, such as FCC couplings. From a theoretical point of view, they are sensitive to different new Physics. From an experimental point of view, they have different signatures and different systematics [7]. Hence, they should be separately measured experimentally. Furthermore, as a proton-antiproton $(p \bar{p})$ collider, Tevatron offers a special chance to measure the amount of (direct) $\mathrm{CP}$-violation in top quark system by measuring the asymmetry in the inclusive single-top versus single-antitop production rates [8]:

$$
A_{t}^{C P}=\frac{\sigma(p \bar{p} \rightarrow t X)-\sigma(p \bar{p} \rightarrow \bar{t} X)}{\sigma(p \bar{p} \rightarrow t X)+\sigma(p \bar{p} \rightarrow \bar{t} X)} .
$$

This is because under the CP symmetry, the initial hadron state $(p \bar{p})$ is invariant. This is the unique opportunity at Tevatron that the LHC cannot offer. To probe CP-violation in top quark interaction at the LHC, one has to measure the CP-violating (more generally, time-reversal violating) observables that make use of the spin information of the produced (anti)top quarks, such as measuring the expectation values of $\left\langle\vec{s}_{t} \cdot \vec{p}_{b} \times \vec{p}_{\ell^{+}}>\right.$and $\left\langle\vec{s}_{\vec{t}} \cdot \vec{p}_{\vec{b}} \times \vec{p}_{\ell^{-}}>\right.$from the decay of top and anti-top, respectively, in the single-top and single-antitop events [8]. Needless to say that CP-violation can also be tested at the LHC in the $t \bar{t}$ events by comparing the production rates of $t_{L} \bar{t}_{L}$ and $t_{R} \bar{t}_{R}$ events, for under the CP operation, a left-handed top $\left(t_{L}\right)$ becomes a right-handed anti-top $\left(\bar{t}_{R}\right)$. One way to 
measure this asymmetry is to detect the asymmetry in the energy distributions of $\ell^{+}$versus $\ell^{-}$in the inclusive $t \bar{t}$ events [9].

\section{Single-top production and decay at NLO QCD}

In order to reliably compare the theory prediction with experimental data on the production rate of the single-top events and the distributions of its final state particles, a next-to-leading order (NLO) QCD calculation has been performed for the s- and t-channel single-top processes [10]. In Ref. [10], we separated the single-top processes into a few smaller gauge invariant sets to organize our calculations, which include corrections to the initial state, final state and top decay in the schannel process, and corrections to the light quark line, heavy quark line and top decay in the t-channel process. Keeping track on each individual contributions is useful for comparing event generators with exact NLO predictions. One can also study the effect from, for example, having correct implementation of top quark spin correlation between its production and decay at the full NLO in QCD.

Given the small single-top production rate at the Tevatron, as predicted by the SM, it is important to study the acceptance of the signal event after imposing the necessary kinematic cuts for detecting them experimentally. We found that the signal acceptances are sensitive to the kinematics cuts. A large $R$-separation cut reduces acceptances significantly because $\Delta R(\ell j)$ is typically less than 1. With tight kinematic cuts, leading order (LO) and NLO acceptances are almost the same, but, with lose cuts, they are quite different. Hence, in order to maximize the signal acceptance, we must impose lose cuts and consequently the NLO acceptance of the signal events cannot be correctly modelled by a scaled-up (multiplied by the $K$-factor extracted from inclusive rate calculations) tree level LO calculation.

To identify the single-top signal events and to test the polarization of top quark, by studying spin correlations among the final state particles, we need to reconstruct the top quark in the singletop events. To do so, we shall first identify the $b$-jet and reconstruct the $W$ boson from top decay. In Table 1, we show the efficiency of finding the correct $b$-jet $\left(\varepsilon_{b}\right)$ in two different algorithms: best-jet algorithm and leading $b$-tagged jet algorithm. The "best-jet" is defined to be the $b$-tagged jet which gives an invariant mass closest to the true top mass when it is combined with the reconstructed $W$ boson after determining the longitudinal momentum $p_{z}^{v}$ of the neutrino from $W$ decay. The leading $b$-tagged jet algorithm picks the leading $b$-tagged jet as the correct $b$-jet to reconstruct the top quark after combining with the reconstructed $W$ boson. As shown in Refs. [11, 12], we find that the best-jet algorithm shows a higher efficiency (about 80\%) in picking up the correct $b$-jet than the leading-jet algorithm (about 55\%) for the $s$-channel single-top events. On the other hand, for the $t$-channel single-top events, the leading $b$-tagged jet algorithm picks up the correct $b$-jet with a higher efficiency, about $95 \%$ for inclusive 2 -jet events and $90 \%$ for exclusive 3 -jet events., cf. Table 1. The reason that the leading $b$-tagged jet algorithm works well in the exclusive 3 -jet $t$-channel single-top events is due to the distinct kinematic difference between $b$ and $\bar{b}$-jets. To reconstruct the top quark in the signal events, we also need to reconstruct the $W$ boson. The $W$ boson is reconstructed with the help of using its mass constraint: $M_{W}^{2}=\left(p_{l}+p_{v}\right)^{2}$. Which one of the two-fold solutions in $p_{z}^{v}$ to be taken depends on the $b$-jet algorithm we used. In the case of best-jet algorithm, we find the one with the smaller magnitude from solving the $W$ mass constraint 


\begin{tabular}{c|c|c|c|c|c|c}
\hline & \multicolumn{3}{|c|}{ best-jet algorithm } & \multicolumn{2}{c}{ leading $b$-tagged jet algorithm } \\
\hline & $s$-channel & \multicolumn{2}{|c}{$t$-channel } & $s$-channel & \multicolumn{2}{c}{$t$-channel } \\
\hline & inclusive 2-jet & inclusive 2-jet & exclusive 3-jet & inclusive 2-jet & inclusive 2-jet & exclusive 3-jet \\
\hline$\varepsilon_{b}$ & $80 \%$ & $80 \%$ & $72 \%$ & $55 \%$ & $95 \%$ & $90 \%$ \\
\hline$\varepsilon_{v}$ & \multicolumn{3}{|c|}{$70 \%$} & & & $84 \%$ \\
\end{tabular}

Table 1: Efficiencies of identifying correct $b$-jet $\left(\varepsilon_{b}\right)$ and picking up correct $p_{z}^{v}\left(\varepsilon_{v}\right)$ in both best-jet algorithm and leading-jet algorithm.

give the best efficiency in $W$ boson reconstruction. In the case of leading $b$-tagged jet algorithm, we use the top quark mass constraint $M_{t}^{2}=\left(p_{b}+p_{l}+p_{v}\right)^{2}$ to pick up the best $p_{z}^{v}$ value. The efficiency for picking up the correct $p_{z}^{v}$ value $\left(\varepsilon_{v}\right)$, at LO and NLO, respectively, is presented in Table 1.

After reconstructing the top quark, one can study the effect of NLO QCD corrections to the measurement of top quark polarization. We found that higher order QCD corrections blur the spin correlation effect. Furthermore, the apparent advantage of some polarization basis at the parton level is washed away after modelling the reconstruction of the top quark as described above.

\section{1 s-channel single-top events and Higgs search}

The $s$-channel single top quark process also contributes as one of the major backgrounds to the SM Higgs searching channel $q \bar{q} \rightarrow W H$ with $H \rightarrow b \bar{b}$. In this case it is particularly important to understand how the $O\left(\alpha_{s}\right)$ corrections change distributions around the Higgs mass region. Because of the scalar property of the Higgs boson, its decay products $b$ and $\bar{b}$ have symmetric distributions. Fig. 1 shows the invariant mass distribution of the ( $b$-jet, $\bar{b}$-jet) system. For a Higgs signal, this invariant mass of the two reconstructed $b$-tagged jets would correspond to a plot of the reconstructed Higgs mass. Thus, understanding this invariant mass distribution will be important to reach the highest sensitivity for Higgs boson searches at the Tevatron. The figure shows that at $O\left(\alpha_{s}\right)$, the invariant mass distribution not only peaks at lower values than at Born level, it also drops off faster. This change in shape is particularly relevant in the region focused on by SM Higgs boson searches of $80 \mathrm{GeV} \leq m_{b \bar{b}} \leq 140 \mathrm{GeV}$ which is also at the $f b$ level. In particular, the NLO contribution from the decay of top quark, while small in its overall rate, has a sizable effect in this region of the invariant mass and will thus have to be considered in order to make reliable background predictions for the Higgs boson searches.

Other kinematic distributions are also changing in shape when going from Born-level to $O\left(\alpha_{s}\right)$. In Ref. [11], we also showed the distribution of $\cos \theta$ for the two $b$-tagged jets, where $\theta$ is the angle between the direction of a $b$-tagged jet and the direction of the ( $b$-jet, $\bar{b}$-jet) system, in the rest frame of the ( $b$-jet, $\bar{b}$-jet) system. Experiments cannot distinguish between the $b$-and the $\bar{b}$-jets, we therefore include both the $b$-jet and the $\bar{b}$-jet in the graph. This distribution is generally flat at Bornlevel, with a drop-off at high $\cos \theta$ due to jet clustering effects, and a drop-off at negative $\cos \theta$ due to kinematic selection cuts. The $O\left(\alpha_{s}\right)$ corrections change this distribution significantly and result in a more forward peak of the distribution, similar to what is expected in Higgs boson production. In other words, a flatter $\cos \theta$ distribution in the s-channel single-top events make it more difficult to separate the $W H$ events from the s-channel single-top events in experimental analysis. 

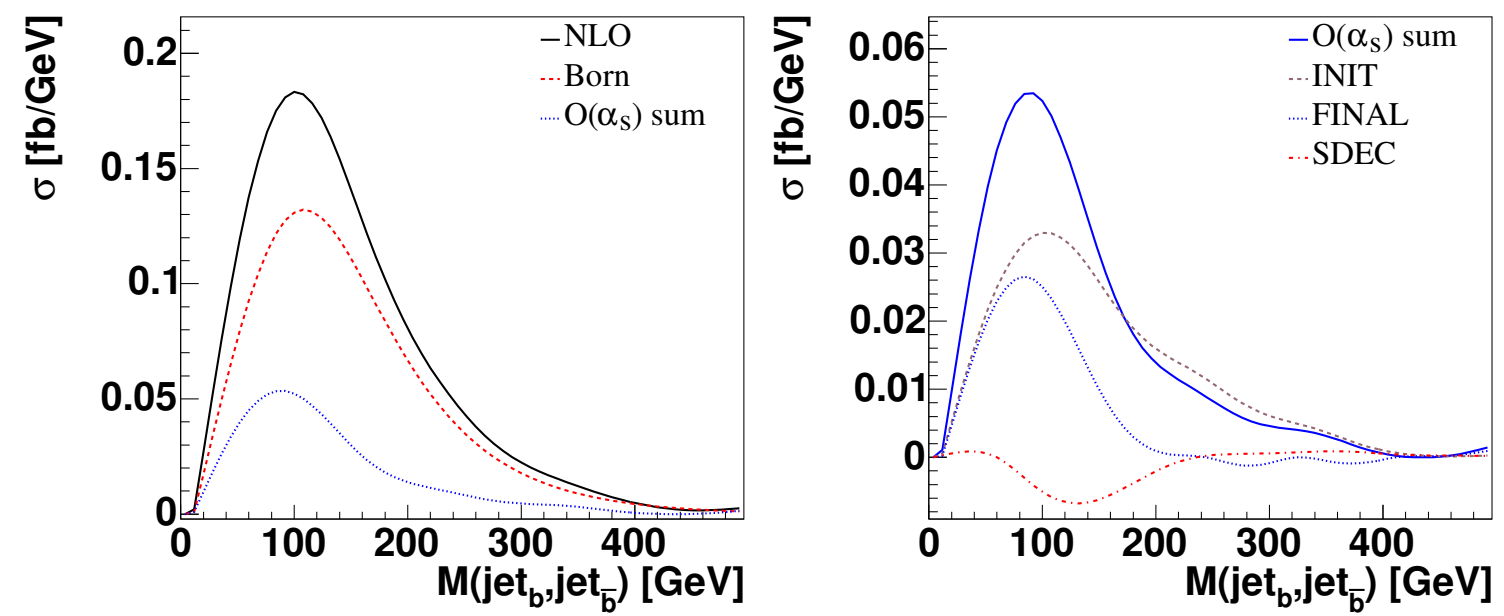

Figure 1: Invariant mass of the ( $b$-jet, $\bar{b}$-jet) system after selection cuts, comparing Born-level to $O\left(\alpha_{s}\right)$ corrections. In the legend, INIT, FINAL and SDEC denotes the contributions from initial state, final state and top quark decay corrections, respectively.

\section{2 t-channel single-top events and Higgs search}

The unique signature of the $t$-channel single top process is the spectator jet in the forward direction, which can be utilized to suppress the copious SM backgrounds, such as $W b \bar{b}$ and $t \bar{t}$ events [13]. Studying the kinematics of this spectator jet is important to have a better prediction of the acceptance of $t$-channel single top quark events. The impact of the NLO QCD corrections on the kinematic properties of the spectator jet has been reported in Ref. [12] for the Tevatron Run-II phenomenology. We found that the NLO QCD corrections to the light-quark (LIGHT) and heavy-quark (HEAVY) lines show almost opposite behavior in the rapidity distribution of the spectator jet. LIGHT shifts the spectator jet to the forward direction while HEAVY shifts it to the central region, and the NLO corrections originated from the decay of top quark does not modify the rapidity distribution of the spectator jet.

One of the most important tasks at the LHC is to find the Higgs boson, denoted as $H$. It has been shown extensively in the literature that the production mechanism of Higgs boson via weak gauge boson fusion is an important channel for the search of Higgs boson. Furthermore, to test whether it is a SM Higgs boson after its discovery, one needs to determine the coupling of $\mathrm{H}-\mathrm{V}$ $V$, where $V$ denotes either $W^{ \pm}$or $Z$, by measuring the production rate of $q \bar{q}(V V) \rightarrow H q^{\prime} \bar{q}^{\prime}$ via weak boson fusion processes. In order to suppress its large background rates, one usual trick is to tag on the two forward-jets resulted from emitting vector boson $V$ to produce Higgs boson via $V V \rightarrow H$. Prior to the discovery of Higgs boson, one can learn about the detection efficiency of the forward jet from studying the $t$-channel single-top process. This is because in the $t$-channel single-top process, the forward jet also results from emitting a $W$ boson which interacts with the $b$ quark from the other hadron beam to produce the heavy top quark. As pointed out in Ref. [13], in the effective- $W$ approximation, a high-energy $t$-channel single top quark event is dominated by a longitudinal $W$ boson and the $b$ quark fusion diagram. It is the same effective longitudinal $W$ boson that dominates the production of a heavy Higgs boson at high energy colliders via the $W$-boson 
fusion process. For a heavy SM Higgs boson, the longitudinal $W$ boson fusion process dominates the Higgs boson production rate. Hence, it is important to study the kinematics of the spectator jet in $t$-channel single top quark events in order to have a better prediction for the kinematics of Higgs boson events via the $W W$ fusion process at the LHC.

\section{General Analysis of $t-b-W$ couplings}

As discussed above, the $t-b$ - $W$ couplings can affect the spin correlations among the decay particles of (anti)top quarks produced in the $t \bar{t}$ events and the production cross section of the singletop events. Therefore, it is desirable to find a systematic way to analyze both $t \bar{t}$ and single-top experimental data to extract out the information on the $t-b-W$ couplings. This was performed in Ref. [6], in which the most general formulation of the $t-b-W$ couplings in any weak-scale effective theory was formulated. It also summarized what we have learned from indirect measurements whose conclusions unavoidably depend on the specific assumption made about the underlying new physics models. The most interesting question to ask is "How to directly measure $t-b-W$ couplings at the Tevatron and the LHC?" as well as "How to distinguish models of EWSB from the results of these direct measurements?".

In the language of electroweak chiral Lagrangian, there are in general 8 different operators at the weak scale to describe the $t-b-W$ couplings which do not require either $t, b$ or $W$ to be on their mass-shells. For on-shell $t$ and $b$ quarks, the above 8 operators reduce to 6 independent ones. Since we will consider only the case that the $W$ boson in the $t-b-W$ couplings couples to massless fermions (quarks or leptons), the number of independent operators is reduced to 4 . In the Unitary gauge, it reads as [3]

$$
\mathscr{L}_{\mathrm{t} b W}=\frac{g}{\sqrt{2}} W_{\mu}^{-} \bar{b} \gamma^{\mu}\left(f_{1}^{L} P_{L}+f_{1}^{R} P_{R}\right) t-\frac{g}{\sqrt{2} M_{W}} \partial_{\nu} W_{\mu}^{-} \bar{b} \sigma^{\mu \nu}\left(f_{2}^{L} P_{L}+f_{2}^{R} P_{R}\right) t+\text { h.c. }
$$

At tree level, the SM predicts $f_{1}^{L}=V_{t b} \simeq 1$, and $f_{1}^{R}=f_{2}^{L}=f_{2}^{R}=0$. Since these form factors can take different values depending on the underlying new physics models that lead to the above effective Lagrangian at the weak scale, we proposed a general analysis to determine these four independent $t-b-W$ couplings. The idea is to use four experimental observables to determine the four independent form factors $f_{1,2}^{L, R}$. They are the experimental measurements on the degrees of longitudinal $\left(f_{0}\right)$ and left-handed $\left(f_{-}\right)$polarizations of the $W$ bosons from top decays measured in the $t \bar{t}$ events and the s- $\left(\sigma_{s}\right)$ and t-channel $\left(\sigma_{t}\right)$ single-top production rates.

In summary, the above four observables can be expressed in terms of the effective $t-b-W$ couplings as: ${ }^{1}$

$$
\begin{aligned}
f_{0} & =\frac{a_{t}^{2}\left(1+x_{0}\right)}{a_{t}^{2}\left(1+x_{0}\right)+2\left(1+x_{m}+x_{p}\right)}, & f_{-} & =\frac{2\left(1+x_{m}\right)}{a_{t}^{2}\left(1+x_{0}\right)+2\left(1+x_{m}+x_{p}\right)}, \\
\Delta \sigma_{t} & =a_{0} x_{0}+a_{m} x_{m}+a_{p} x_{p}+a_{5} x_{5}, & \Delta \sigma_{s} & =b_{0} x_{0}+b_{m} x_{m}+b_{p} x_{p}+b_{5} x_{5},
\end{aligned}
$$

where $\Delta \sigma$ stands for the variation from the SM NLO prediction, and the degree of right-handed polarization of the $W$ boson from top decay is obtained from $f_{+}=1-f_{-}-f_{0}$. The numerical

\footnotetext{
${ }^{1}$ There are typos in Eqs. (5) and (6) of Ref. [6], in which $x_{t}$ should be $a_{t}$.
} 
values of the $a_{i}$ and $b_{i}$ coefficients were given in Ref. [6] for the Tevatron and the LHC, respectively. They were obtained by integrating over the parton luminosities which are evaluated using CTEQ6L1 parton distribution functions. Furthermore, in the above equations,

$$
\begin{aligned}
& x_{0}=\left(f_{1}^{L}+f_{2}^{R} / a_{t}\right)^{2}+\left(f_{1}^{R}+f_{2}^{L} / a_{t}\right)^{2}-1, \quad x_{m}=\left(f_{1}^{L}+a_{t} f_{2}^{R}\right)^{2}-1, \\
& x_{p}=\left(f_{1}^{R}+a_{t} f_{2}^{L}\right)^{2}, \quad x_{5}=a_{t}^{2}\left(f_{2}^{L^{2}}+f_{2}^{R^{2}}\right), \quad \text { with } \quad a_{t}=m_{t} / M_{W} .
\end{aligned}
$$

In case that a new light resonance, either a scalar or vector boson, is found, the s-channel process could be significantly enhanced and its production rate may not be dominated by a virtual $W$-boson (s-channel) diagram. The above formulas may also apply to models with extra heavy fermion ( $T$ ), such as the top quark partner in the Little Higgs (LH) Models that couples to the SM $b$ quark and $W$ boson. The $T b W$ coupling in general has the same form of the general $t b W$ coupling given above, and the expressions for single- $T$ production cross sections are exactly the same as single-top except for the heavy mass $m_{T}$. The size of the coefficients in the production cross sections decrease drastically with a greater mass $m_{T}$. For instance, at $m_{T}=500 \mathrm{GeV}$ the $a_{0}$ coefficient decreases one order of magnitude with respect to the value for $m_{T}=178 \mathrm{GeV}$ at the LHC. Furthermore, in the t-channel single- $T$ process, the $a_{0}$ coefficient, corresponding to longitudinal $W$ boson contribution, dominates its production cross section. This is in analogy with the SM t-channel single-top production. in which longitudinal $W$ boson contribution dominates the inclusive cross section.

Finally, we note that with enough precision in determining the four individual $t b W$ couplings at the LHC, we could start distinguishing models of EWSB [6], such as the MSSM and the Technicolor assisted Topcolor (TC2) mode.

\section{Top quark and EWSB}

\subsection{MSSM and TC2}

With its mass around the weak scale, top quark could very well be a special quark that might play an important role in the EWSB. The bottom-up approach is to construct the most general electroweak chiral Lagrangian to describe the interaction of top quark and then compare the effective theory predictions with experimental data to extract out information on the coefficients of the relevant effective operators. Since these coefficients depend on the underlying new physics models, it is possible to discriminate models of EWSB from their values. Usually, two classes of EWSB models are considered in the literature: weakly interacting models and strongly interacting models. The former consists of elementary Higgs boson(s) that originate from spontaneously symmetry breaking (such as MSSM and LH models), and the latter may predict composite Higgs boson(s) due to some strongly interacting dynamical symmetry breaking mechanism (such as TC2 models). In the MSSM, the EWSB is generated radiatively due to heavy top quark contribution in loops. In the TC2 model, top quark condensate relates the heavy top quark mass with the weak scale $(v \sim 246 \mathrm{GeV})$ at which the $W$ and $Z$ bosons become massive gauge bosons. It is interesting to note that numerically $m_{t} \sim v / \sqrt{2} \sim M_{W}+M_{Z}$.

Because bottom quark is the isospin partner of top quark, its interactions can also be sensitive to new physics models of EWSB. For example, in the MSSM, two Higgs doublets are required by 

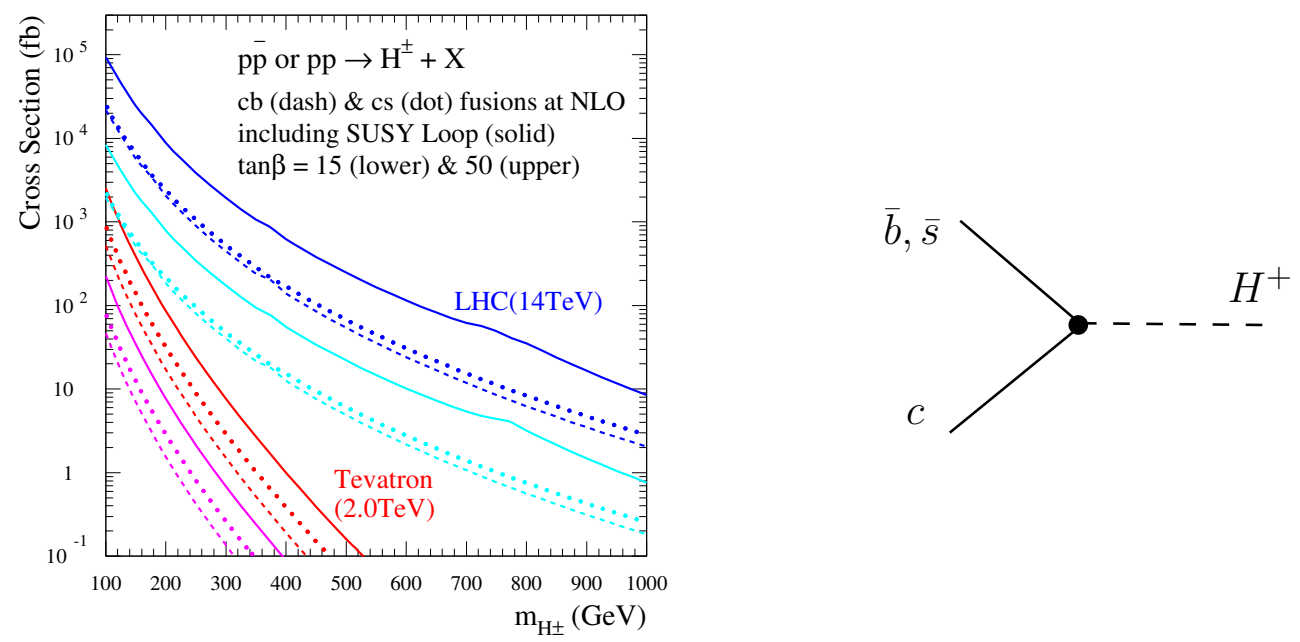

Figure 2: The MSSM $H^{ \pm}$production via $c b$ (and $c s$ ) fusions at the Tevatron and the LHC.

supersymmetry. When $\tan \beta$ (the ratio of vacuum expectation values of the two Higgs doublets) is large, the bottom quark Yukawa coupling can become large enough that the production rates of $b \bar{b} H$ events can become measurable at the Tevatron and the LHC [14]. Assuming a horizontal $\mathrm{U}(1)$ flavor symmetry in the soft-breaking sector of MSSM for generating the scalar quark masses, the (32) and (23) entries of the trilinear coupling matrix $A_{u}$ for up-squarks can have the same size as the (33) entry of $A_{u}$ and lead to large flavor mixing between stop ( $\left.\tilde{t}\right)$ and scharm ( $(\tilde{c})$ [15]. As shown in Ref. [15], large $\tilde{t}-\tilde{c}$ mixing can enhance the s-channel charged Higgs boson production via $c \bar{b} \rightarrow H^{+}$, cf. Fig. 2, as well as the FCNC decay process $h^{0} \rightarrow t \bar{c}$. We note that the s-channel $H^{+}$production can be tested in the single-top events when $H^{+}$decays into a pair of $b$ and $W$ at the Tevatron and the LHC. The decay branching ratio $\mathrm{BR}\left(h^{0} \rightarrow t \bar{c}\right)$ can range from $10^{-5}$ to $10^{-3}$ and is sensitive to the mass of the lightest stop and the mixing of stop and scharm. Furthermore, the chirality of $H^{+}-b-c$ couplings can be determined at future photon-photon linear collider via $\gamma \gamma \rightarrow(b \bar{b})(c \bar{c}) \rightarrow H^{+} b \bar{c}$ by polarizing the polarization of the incoming photon beams [16].

Similarly, in the TC2 model, top-pion $\left(\pi^{\prime}\right)$ can be produced at resonance via the s-channel process $c \bar{b} \rightarrow \pi^{\prime} \rightarrow t \bar{b}$ due to the presence of a large $t_{R}-c_{R}$ mixing that is consistent with all the existing FCNC data [17]. We note that the CKM matrix is determined by the product of the lefthanded up- and down-type quark rotation matrices (to diagonalize the quark mass matrices) and the right-handed rotation matrices are not fixed by the CKM matrix. Hence, a large $t_{R}-c_{R}$ mixing does not contradict with the existing experimental data. In the Topflavor model [18], the production of an s-channel $W^{\prime}$ heavy boson can also produce single-top signature through $q \bar{q}^{\prime} \rightarrow W^{\prime} \rightarrow t \bar{b}$ at hadron colliders.

In summary, carefully studying the interactions of top quark in experimental data can help distinguishing models of EWSB. A few examples are given in Table 2 to illustrate the idea.

\subsection{Little Higgs model}

In the Little Higgs model, the Higgs boson mass is naturally at the weak scale, because large quadratic correction to the Higgs boson mass term induced by the top quark loop is cancelled by the fermionic partner $(T)$ of top quark $(t)$ due to the approximate global symmetry which relates 


\begin{tabular}{c|c|c}
\hline model & top Yukawa coupling & bottom Yukawa coupling \\
\hline SM & $\sim 1$ & $\sim 1 / 40$ \\
\hline MSSM $(\tan \beta=40)$ & $\sim 1 / 40$ & $\sim 1$ \\
\hline TC2 & $\sim 1$ & $\sim 1$ \\
\hline
\end{tabular}

Table 2: Discriminate models of EWSB by testing the interaction of top and bottom quarks to Higgs boson.

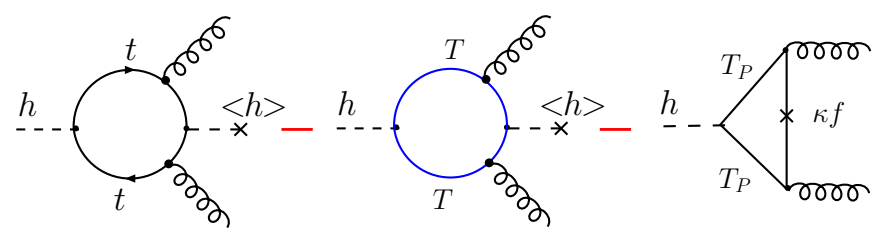

Figure 3: Representative Feynman diagrams for $g g \rightarrow h$ production.

T with t (i.e., Little Higgs mechanism) [19]. Furthermore, to ensure $\rho$-parameter to be one at tree level, a discrete symmetry called T-parity was introduced in the Little Higgs model with T-parity (LHT). Consequently, the effective cutoff scale of the model $\Lambda=4 \pi f$ can be as low as $10 \mathrm{TeV}$ and the masses of new heavy resonances can be of sub-TeV [20]. The LHT model is particularly interesting because it also provides a dark matter candidate which is the lightest T-odd particle $A_{H}$, the heavy bosonic T-partner of photon. Another important feature of this model is that new Higgs couplings are induced in the part of effective theory that generates the masses of the extra heavy T-partner (either T-odd or T-even) fermions needed for protecting the Higgs boson mass at the weak scale. In Ref. [21] we showed that these new Higgs couplings can lead to non-decoupling effect and alter our conclusions on the collider phenomenology of Higgs boson.

For example, the tree level couplings of Higgs boson to weak gauge bosons and fermions are all suppressed relative to their $\mathrm{SM}$ values by a factor $1-c\left(v_{S M}^{2} / f^{2}\right)$ where $v_{S M} \sim 246 \mathrm{GeV}$ and the coefficient $c$ depends on the specific coupling and model scenario. In Fig. 3, we show some representative Feynman diagrams contributing to the production process $g g \rightarrow h$. We found that the production rate of Higgs boson via gluon-gluon fusion is also suppressed relative to the SM rate. This can be understood as follows. In the Littlest Higgs model [22], the contribution from the T-partner $(T)$ of top quark partially cancel the top quark loop contribution, similar to the effect of cancelling the quadratic divergencies in Higgs boson mass correction. The additional contribution induced by the T-odd heavy fermions further suppress the production rate of $g g \rightarrow h$ due to the nondecoupling effect originated from the mass generation mechanism for those heavy T-odd fermions [21]. With $f=700 \mathrm{GeV}$, which is consistent with low energy precision data, the cross section $\sigma(g g \rightarrow h)$ can be reduced by about $35 \%$ for $m_{h}$ around $115 \mathrm{GeV}$. It is important to note that the total decay width of Higgs boson in the LHT is always smaller than that predicted by the SM, for the cancellation of quadratic divergencies in Higgs boson mass corrections are among particles with the same spin statistics. While the partial decay width of $\Gamma(h \rightarrow \gamma \gamma)$ does not change very much from the SM prediction, the decay branching ratio $\mathrm{BR}(h \rightarrow \gamma \gamma)$ can increase by as much as $30 \%$ for the total decay width of Higgs boson is largely reduced by the smaller bottom quark Yukawa coupling. Consequently, the discovery potential of the LHC for a light Higgs boson with 


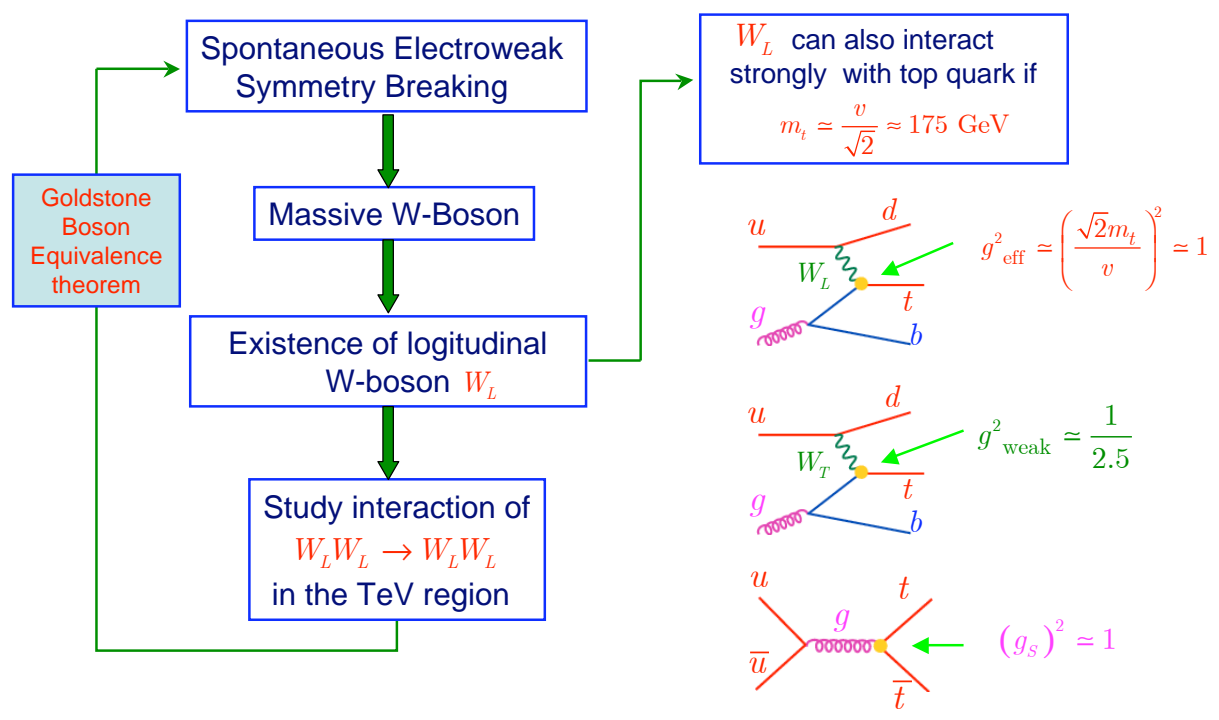

Figure 4: Schematical diagrams to show the motivation of study done in Ref. [13] for t-channel single-top production.

mass around $100 \mathrm{GeV}$ can be dramatically altered from its usual conclusion. For instance, the rate of $g g \rightarrow h \rightarrow \gamma \gamma$ can be reduced by about $14 \%$, while the $W$-boson fusion rate increases by about $22 \%$ as compared to the SM prediction, cf. Table 1 of Ref. [21]. Hence, in the LHT model, the $W$-boson fusion process becomes the main discovery channel of a $100 \mathrm{GeV}$ Higgs boson at the LHC [21].

\subsection{Higgsless model}

If Higgs boson exists, discovering the Higgs boson and studying its interaction is essential to probe the electroweak symmetry breaking and the flavor symmetry breaking. Otherwise, we need to carefully study the interaction among longitudinal $W$ (representing $W^{ \pm}$or $Z$ ) bosons $\left(W_{L}\right)$ in the TeV region [23] as well as the interaction of longitudinal $W$ boson to heavy fermions (top and bottom) [24]. What motivated my 1990 single-top paper was to find a new way to study the interaction of longitudinal $W$ boson to heavy top quark (with its mass around $180 \mathrm{GeV}$ ) [13]. Schematically, the relation between the $W_{L} W_{L} \rightarrow W_{L} W_{L}$ scattering and the t-channel single-top process is shown in Fig. 4.

In the effective- $W$ approximation, the t-channel single-top production is dominated by the interaction of longitudinal $W$ boson $\left(W_{L}\right)$ to heavy top. While the interaction of transverse $W$ boson $\left(W_{T}\right)$ to top quark has the typical weak coupling strength, the interaction of $W_{L}$ and $t$ is effectively of the same strength as $O(1)$ Yukawa coupling of top quark based on the Goldstone Equivalence Theorem [25].

In some models of extra-dimension, say, Higgsless model, there is neither elementary nor composite Higgs boson to regulate the bad high energy behavior of the $W_{L} W_{L} \rightarrow W_{L} W_{L}$ scattering amplitudes in the $\mathrm{TeV}$ region. The breakdown of the unitarity of scattering amplitudes is delayed by the extra heavy Kaluza-Klein gauge bosons predicted in the four-dimensional effective theory 
[26]. In such kind of models, the interaction of top quark (and its partners) with longitudinal $W$ bosons can become more important. Hence, we need to study the production of top quark (and its partners) from $W$-boson fusion process in the $\mathrm{TeV}$ region. It is also possible that the extra bosons needed for delaying the unitarity breakdown in the $W_{L} W_{L} \rightarrow W_{L} W_{L}$ scattering amplitudes can lead to interesting top quark phenomenology. For example, $Z^{\prime}$ can modify the $t \bar{t}$ event distributions and $W^{\prime}$ can induce extra production rate for single-top events at the LHC.

\section{Summary}

Because of its heavy mass, top quark may very well be a special quark that could provide hints on electroweak (and flavor) symmetry breaking mechanism. Though theorists have explored many probable ideas in which top quark plays a crucial role in the construction of the theory model, it is entirely up to the experimental data to tell us which theory model is the closest to true Nature. Needless to say that we need experimental data to advance our acknowledge. Currently, we are waiting for the exciting new discovery of single-top events at the Tevatron Run-II. With the huge production rate of top quarks at the LHC, we are able to study many details about its property. The physics of top quark is indeed very rich, and it is also a sure thing that we will learn much more with the realization of future ILC.

\section{Acknowledgments}

I would like to thank the local organizers for their hospitality and for making the Workshop to be a productive one. I also thank my collaborators in the past years who helped me to discover the beauty in physics of top quark and to invent new ways to study its phenomenology. They are G.L. Kane, G.A. Ladinsky, D.O. Carlson, E. Malkawi, T.M.P. Tait, F. Larios, H.-J. He, L. DiazCruz, S. Mrenna, Q.-H. Cao, K. Tobe, and C.-R. Chen. I apologize that due to the limited space in this write-up, I am not being able to cite all the references in the literature that are relevant to top quark physics, but they can be found collectively in the upcoming TeV4LHC report. This work is supported in part by the U. S. National Science Foundation under award PHY-0244919.

\section{References}

[1] C. Quigg, Top-ology, Phys. Today 50N5 (1997) 20.

[2] CDF and D0 Collaborations, Precision Measurement of Top QUark Mass in Dilepton Channel, preprint no. Fermilab-conf-06-030-E, Jan 2006.

[3] G.L. Kane, G.A. Ladinsky, and C.-P. Yuan, Using the Top Quark for Testing Standard Model Polarization and CP Predictions, Phys. Rev. D45 (1992) 124.

[4] A. Juste, Top Quark Physics at the ILC, talk given at Top Quark Symposium, Ann Arbor, April 7-8, 2005.

[5] S. Mrenna and C.-P. Yuan, QCD Radiative Decay of the Top Quark Produced in Hadron Collisions, Phys. Rev. D46 (1992) 1007.

[6] C.-R. Chen, F. Larios and C.-P. Yuan, General Analysis of Single Top Production and W Helicity in Top Decay, Phys. Lett. B631 (2005) 126. 
[7] T.M.P. Tait and C.-P. Yuan, Single Top Quark Production as a Window to Physics Beyond the Standard Model, Phys. Rev. D63 (2001) 014018.

[8] C.-P. Yuan, Strategies for Probing CP Properties in the Top Quark System, Mod. Phys. Lett. A10, No. 8 (1995) 627.

[9] C.R. Schmidt, M.E. Peskin, A Probe Of Cp Violation In Top Quark Pair Production At Hadron Supercolliders, Phys. Rev. Lett. 69 (1992) 410.

[10] Q.-H. Cao and C.-P. Yuan, Single top quark production and decay at next-to-leading order in hadron collision, Phys. Rev. D71 (2005) 054022.

[11] Q.-H. Cao, R. Schwienhorst and C.-P. Yuan, Next-To-Leading Order Corrections to Single Top Quark Production and Decay at Tevatron. 1. S-Channel Process, Phys. Rev. D71 (2005) 054023.

[12] Q.-H. Cao, R. Schwienhorst, R. Brock, J. Benitez and C.-P. Yuan, Next-To-Leading Order Corrections to Single Top Quark Production and Decay at Tevatron. 2. t-Channel Process, Phys. Rev. D72 (2005) 094027.

[13] C.-P. Yuan, A New Method to Detect a Heavy Top Quark at the Fermilab Tevatron, Phys. Rev. D41 (1990) 42.

[14] J.L. Diaz-Cruz, H.-J. He, T. Tait, and C.-P. Yuan, Higgs Bosons with Large Bottom Yukawa Coupling at Tevatron and LHC, Phys. Rev. Lett. 80 (1998) 4641.

[15] J.L. Diaz-Cruz, H.-J. He and C.-P. Yuan, Soft SUSY Breaking, Stop-Scharm mixing and Higgs Signatures, Phys. Lett. B530 (2002) 179.

[16] H.-J. He, S. Kanemura and C.-P. Yuan, Determining the Chirality of Yukawa Couplings via Single-Charged Higgs Boson Production in Polarized Photon Collision, Phys. Rev. Lett. 89 (2002) 101803.

[17] H.-J. He and C.-P. Yuan, New Method for Detecting Charged Pseudoscalars at Colliders, Phys. Rev. Lett. 83 (1999) 28.

[18] E. Malkawi, T. Tait, and C.-P. Yuan, A Model of Strong Flavor Dynamics for the Top Quark, Phys. Lett. B385 (1996) 304.

[19] N. Arkani-Hamed, A.G. Cohen and H. Georgi, Electroweak symmetry breaking from dimensional deconstruction, Phys. Lett. B513 (2001) 232.

[20] H. C. Cheng and I. Low, TeV symmetry and the Little hierarchy problem, JHEP 0309 (2003) 051.

[21] C.-R. Chen, K. Tobe and C.-P. Yuan, Higgs Boson Production And Decay In Little Higgs Models With T-Parity, e-Print Archive: [hep-ph/0602211].

[22] N. Arkani-Hamed, A.G. Cohen, E. Katz and A.E. Nelson, The Littlest Higgs, JHEP 0207 (2002) 034.

[23] Jon Bagger, et. al., LHC Analysis of the Strongly Interacting WW System: Gold-Plated Modes, Phys. Rev. D52 (1995) 3878.

[24] F. Larios, T. Tait and C.-P. Yuan, Anomalous $W^{+} W^{-} t \bar{t}$ Couplings at the $e^{-} e^{+}$Linear Collider, Phys. Rev. D57 (1998) 3106.

[25] H.-J. He, Y.-P. Kuang, and C.-P. yuan, Equivalence Theorem and Probing the Electroweak Symmetry Breaking Sector, Phys. Rev. D51 (1995) 6463.

[26] C. Csaki, C. Grojean, L. Pilo, and J. Terning, Towards A Realistic Model Of Higgsless Electroweak Symmetry Breaking, Phys. Rev. Lett 92 (2004) 101802. 